\title{
Correction: Leveraging Google Trends to investigate the global public interest in rheumatoid arthritis
}

\author{
Guo-Cui Wu ${ }^{1} \cdot$ Sha-Sha Tao ${ }^{2,3} \cdot$ Chan-Na Zhao ${ }^{2,3} \cdot$ Yan-Mei Mao ${ }^{2,3} \cdot$ Qian Wu ${ }^{2,3} \cdot{\text { Yi-Lin } \text { Dan }^{2,3} \cdot \text { Hai-Feng Pan }}^{2,3}$
}

Received: 28 May 2019 / Accepted: 4 June 2019 / Published online: 6 June 2019

c) Springer-Verlag GmbH Germany, part of Springer Nature 2019

Keywords Google Trends · Global public interest $\cdot$ Rheumatoid arthritis · Seasonality

Dear Editor,

We are sorry for copying several sentences in our Methods section [1]. Since it is our first study on Google Trends, we are not skilled in describing the method of Google Trends analysis. This is why, we refer to previously published methods and copy-related text verbatim [2,3]. Due to the same study period, design, and statistical analyses, there might be an overlap between Methods section of our and previously published articles. Having mentioned that, we confirm that the idea, results, and conclusion of our own study are authentic and not copied from elsewhere.

We accept that copying sentences and paragraphs from published articles is inappropriate, and is mainly due to inadequate English writing skills. Our article was written without any external language editing support.

Hai-Feng Pan

panhaifeng@ahmu.edu.cn; panhaifeng1982@sina.com

1 School of Nursing, Anhui Medical University, 15 Feicui Road, Hefei, Anhui, People's Republic of China

2 Department of Epidemiology and Biostatistics, School of Public Health, Anhui Medical University, 81 Meishan Road, Hefei 230032, Anhui, People's Republic of China

3 Anhui Province Key Laboratory of Major Autoimmune Diseases, 81 Meishan Road, Hefei, Anhui,

People's Republic of China

\section{Compliance with ethical standards}

Conflict of interest All the authors declared no conflict of interest.

\section{References}

1. Wu GC, Tao SS, Zhao CN, Mao YM, Wu Q, Dan YL, Pan HF (2019) Leveraging Google Trends to investigate the global public interest in rheumatoid arthritis. Rheumatol Int. https://doi. org/10.1007/s00296-019-04297-6

2. Kardeş S (2019) Seasonal variation in the internet searches for gout: an ecological study. Clin Rheumatol 38(3):769-775. https ://doi.org/10.1007/s10067-018-4345-2

3. Jellison SS, Bibens M, Checketts J, Vassar M (2018) Using Google Trends to assess global public interest in osteoarthritis. Rheumatol Int 38(11):2133-2136. https://doi.org/10.1007/s0029 6-018-4158-2

Publisher's Note Springer Nature remains neutral with regard to jurisdictional claims in published maps and institutional affiliations. 